\title{
Um procedimento semi-analítico para a análise dinâmica de barragens de concreto
}

\author{
Paulo Marcelo Vieira Ribeiro(1), Lineu José Pedroso ${ }^{(2)}$
}

\begin{abstract}
Resumo
Em um trabalho anterior foi exposto um procedimento simplificado para a análise da interação barragem-reservatório considerando apenas a participação do modo fundamental, com a solução de uma equação de frequências e posterior construção de um modelo numérico equivalente. Este trabalho representa uma continuidade desta linha de pesquisa, explorando pontos de destaque da metodologia proposta, tais como: variação da fonte de excitação, influência do modelo de pressões hidrodinâmicas, contribuição de modos superiores na resposta dinâmica e avaliação de tensões em um instante de tempo arbitrário. Ao final, será apresentado um exemplo de aplicação e discutidas as vantagens e limitações do procedimento proposto.
\end{abstract}

Palavras-chaves: Barragens. Reservatório. Interação Fluido-Estrutura. Semi-Analítico. Dinâmica .

1 Universidade Federal de Pernambuco - UFPE. paulo.vribeiro@ufpe.br

2 Universidade de Brasília - UnB. lineu@unb.br 


\section{Introdução}

A análise dinâmica de barragens submetidas aos efeitos de interação com o reservatório adjacente é um assunto de grande interesse em regiões sismicamente ativas. Soluções numéricas envolvendo ambos os domínios (fluido e estrutura) são geralmente dispendiosas em relação ao tempo de processamento computacional. Adicionalmente, encontram-se limitadas a códigos computacionais específicos, que permitem a análise de problemas acoplados. A geometria tridimensional da estrutura pode ser reduzida a um caso específico bidimensional, no caso de barragens de gravidade. Ainda assim, existem numerosas possibilidades para o perfil típico adotado, tornando uma solução totalmente analítica praticamente impossível, ou aplicada apenas a poucos casos específicos, assim como demonstrado por Lee e Tsai (1991). Uma alternativa surge com o uso de soluções intermediárias, utilizando a precisão de soluções analíticas aliada ao vasto campo de aplicação dos métodos numéricos.

O problema de interação barragem-reservatório já foi analisado por diversos autores. A grande maioria dos trabalhos segue basicamente a mesma linha de desenvolvimento dos artigos clássicos elaborados por Chopra $(1970,1978)$ e seus colaboradores (Fenves e Chopra, 1985). Desde o trabalho pioneiro de Westergaard (1933) verifica-se a importância da inclusão dos efeitos de interação dinâmica com o fluido. A barragem, que inicialmente era suposta rígida, passou a ser tomada como flexível. Em uma etapa seguinte os efeitos de compressibilidade do fluido foram incluídos na análise, sendo tributários da freqüência de vibração do sistema barragem-reservatório. Numerosos procedimentos práticos foram desenvolvidos em seguida, considerando como ponto de partida a identificação do período de vibração incluindo os efeitos de interação, onde os efeitos de absorção da fundação foram acrescentados em uma etapa posterior. O ponto comum a todos esses artigos é exatamente a presença de ábacos de projeto, baseados em uma geometria padrão de barragem, que permitem relacionar o período de vibração no vácuo ao período de vibração acoplado. Como os efeitos de interação são tributários da freqüência acoplada, esta etapa revela-se a mais importante da análise, com o interesse recente de pesquisadores em busca de soluções semi-analíticas para a solução do período de vibração acoplado em geometrias arbitrárias, evitando a utilização das simplificações inerentes aos procedimentos anteriores. A limitação corrente é que mesmo as mais recentes propostas de procedimentos semi-analíticos, tais como Miquel e Bouanaani (2010) e Ribeiro et al. (2009), ainda são limitadas ao problema de interação barragem-reservatório.

Um trabalho anterior (Ribeiro, 2010) revela uma alternativa para a solução simplificada deste tipo de problema empregando uma análise modal auxiliar em elementos finitos da geometria da barragem (Figura 1). Neste caso, são identificados os parâmetros generalizados do modo fundamental (B), que são posteriormente aplicados na solução de uma equação de freqüências (C) para avaliação do período fundamental da estrutura incluindo os efeitos do reservatório. 
O procedimento esquematizado na Figura 1 tem etapas bem definidas e pode ser conduzido sem maiores dificuldades. Neste caso, o objetivo é o cálculo da freqüência fundamental acoplada $(\omega)$, que é aplicada na expressão de massa generalizada produzida pelo fluido (etapa A.1), cuja equação é indicada abaixo.

$$
\widetilde{M}_{\text {fluido }}=\int_{0}^{H} \frac{P(0, y, \omega)}{\bar{A}} \phi(y) d y
$$

onde $\overline{\boldsymbol{A}}$ e $\boldsymbol{H}$ indicam, respectivamente: uma amplitude relacionada a coordenada generalizada em questão e a profundidade do reservatório (ou altura da cavidade acústica).

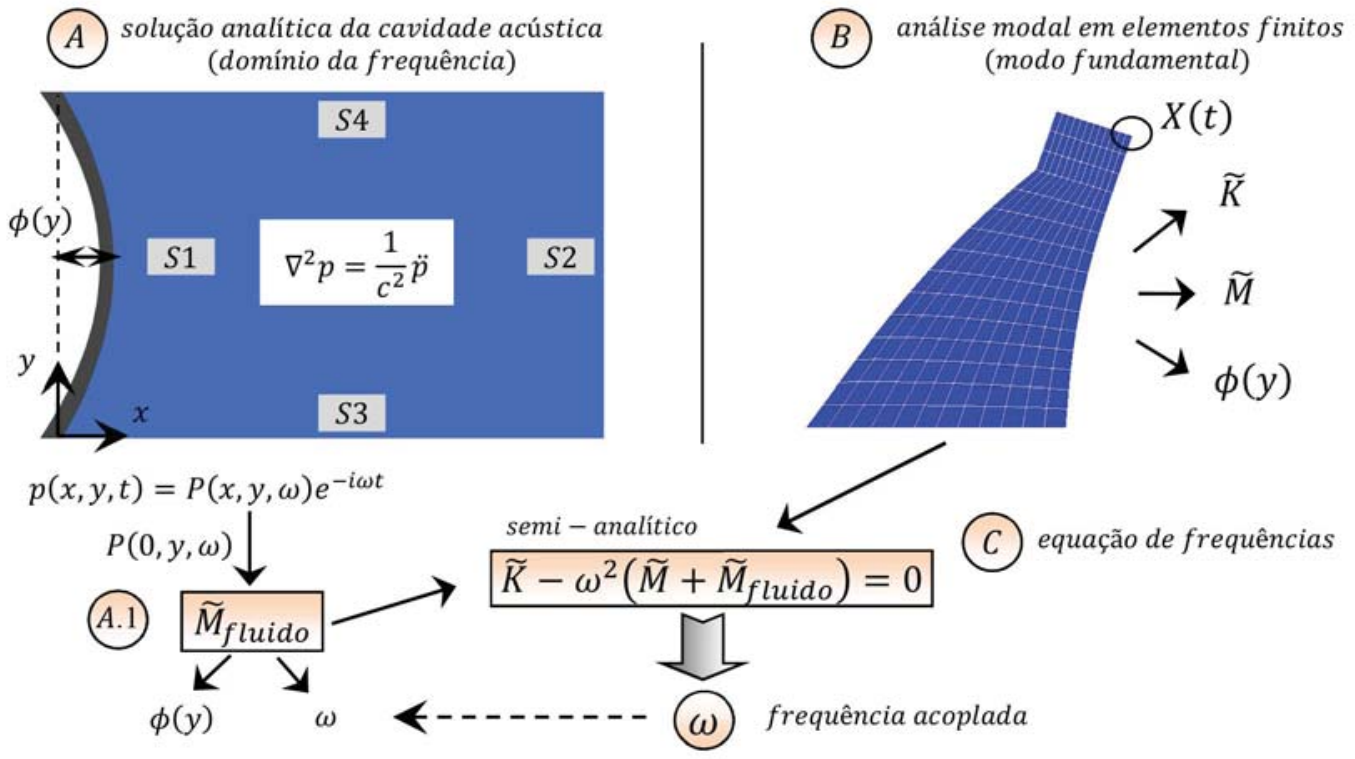

Figura 1: Esquema proposto para a solução semi-analítica (equação de freqüências)

O termo em (1) pode ser acrescentado a uma equação de movimento em função de um parâmetro generalizado $\mathrm{X}(t)$, resultando em equações da estrutura acoplada não-amortecida e amortecida definidas por (2) e (3), respectivamente.

$$
\begin{gathered}
\left(\widetilde{M}+\widetilde{M}_{\text {fluido }}\right) \ddot{X}(t)+\widetilde{K} X(t)=\tilde{F}(t) \\
\left(\widetilde{M}+\widetilde{M}_{\text {fluido }}\right) \ddot{X}(t)+\tilde{C} \dot{X}(t)+\widetilde{K} X(t)=\tilde{F}(t)
\end{gathered}
$$


As expressões anteriores são válidas para uma coordenada generalizada $X(t)$ previamente definida e assumem parâmetros generalizados da estrutura inalterados pela presença do fluido (hipótese do método).

O procedimento acima descrito revela uma estratégia eficaz na eliminação do reservatório, substituindo-o por efeitos equivalentes na interface fluido-estrutura. Assim, o problema pode ser simplificado a solução de uma única equação de equilíbrio dinâmico. É evidente que a solução proposta é válida apenas para o modo fundamental do sistema barragem-reservatório, com todos os parâmetros generalizados consistentes com esta forma modal. Esta representa a principal limitação do procedimento proposto, que será explorado detalhadamente a seguir.

\section{Modelos de cálculo das pressões hidrodinâmicas}

O cálculo da massa generalizada adicional produzida pelo fluido apresenta relação direta com o modelo de pressões hidrodinâmicas adotado. Assim, as condições de contorno indicadas na Figura 1 (etapa A) e a própria equação governante de pressões da cavidade influenciam a parcela de massa adicional do sistema acoplado.

As condições de contorno em uma cavidade com a condição de não-perturbação no limite de um reservatório longitudinal infinito, reflexão total na fundação e efeitos de ondas de superfície livre desprezados são dadas por:

$$
\begin{gathered}
S 2 \rightarrow \lim _{x \rightarrow \infty} p(x, y, t)=0 \\
\left.S 3 \rightarrow \frac{\partial p(x, y, t)}{\partial y}\right|_{y=0}=0 \\
S 4 \rightarrow p(x, H, t)=0
\end{gathered}
$$

Parâmetros adicionais envolvem a consideração ou não da compressibilidade do fluido, além das características da fronteira vibrante (com deslocamento definido por $u$ ), que pode ser tomada como rígido-móvel ou flexível. Assim, resultam os modelos de pressões hidrodinâmicas indicados na Figura 2, que resumem os casos a serem estudados. 


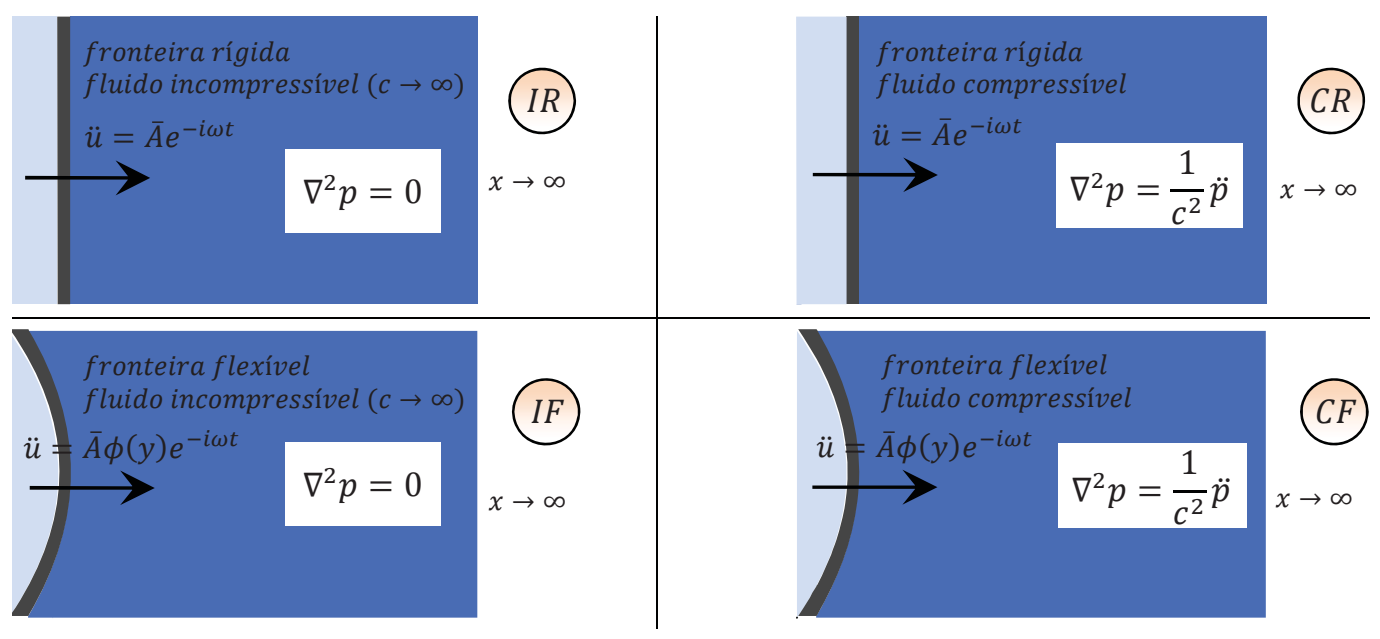

Figura 2. Modelos de pressões hidrodinâmicas

Na Figura 2 as abreviações de cada caso indicam: (I), incompressível, (C) compressível, $(\mathrm{R})$ rígido-móvel e (F) flexível. O caso geral é dado por ( $\mathrm{CF}$ ), cuja solução é indicada pela expressão (7). Os demais casos podem ser obtidos com a redução desta equação (Silva, 2007) ao modelo incompressível $(c \rightarrow \infty)$, rígido-móvel $(\phi=1)$, ou ambos.

$$
P(x, y, \omega)=\frac{2 \rho_{f} \bar{A}}{H} \sum_{n=1}^{\infty} \frac{1}{\sqrt{\hat{\alpha}_{n}}} \int_{0}^{H} \phi(y) \cos \left(\kappa_{n} y\right) d y\left[e^{-\sqrt{\hat{\alpha}_{n}} x}\right] \cos \left(\kappa_{n} y\right) \quad \rightarrow \quad C F
$$

onde:

$$
\begin{gathered}
\hat{\alpha}_{n}=\left(\kappa_{n}\right)^{2}-\frac{\omega^{2}}{c^{2}} \\
\kappa_{n}=\frac{(2 n-1) \pi}{2 H}
\end{gathered}
$$

e $\rho_{f}$ indica a densidade do fluido.

É importante observar que os casos (IF) e (IR) são independentes de ${ }^{\omega}$, eliminando a etapa de solução da equação de frequências (Item C, Figura 1). Assim, a avaliação da massa generalizada adicional produzida pelo fluido pode ser conduzida de forma imediata. 


\section{Ações equivalentes em um sistema fluido-estrutura submetido a uma excitação sísmica}

No caso de uma excitação de crigem sísmica é conveniente analisar o problema em termos de componentes do soln $\left({ }^{u_{g}}\right)$ e relativa $\left({ }^{u_{r}}\right)$, que somadas representam a resposta dinâmica total do sistema $\left({ }^{u_{t}}\right)$. Estes conceitos são esclarecidos na Figura 3, que ilustra um sistema de um grau de liberdade não-amortecido.
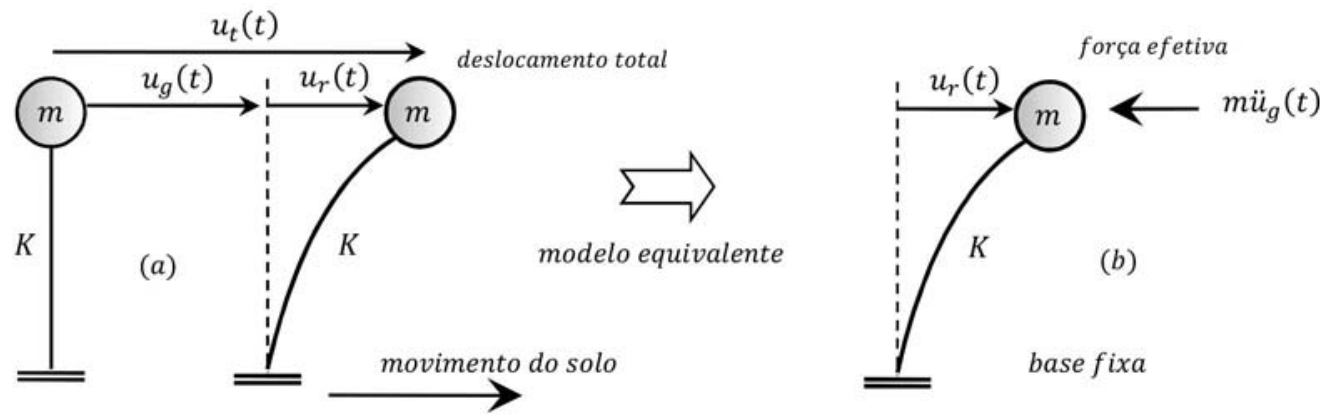

Figura 3. Excitação sísmica de um sistema de 1 grau de liberdade. Sistema desacoplado (a), modelo equivalente (b)

A equação de equilíbrio dinâmico deste sistema é obtida de forma imediata. Assim:

$$
m \ddot{u}_{t}(t)+K u_{r}(t)=0
$$

Da Figura 3a resulta uma aceleração total definida por: : $\ddot{u}_{t}(t)=\ddot{u}_{g}(t)+\ddot{u}_{r}(t)$. Então, substituindo esta última expressão em (10):

$$
m \ddot{u}_{r}(t)+K u_{r}(t)=-m \ddot{u}_{g}(t)=p_{e f}(t)
$$

onde o termo do lado direito de (11) representa a força efetiva $\left(p_{e f}\right)$ gerada pelo sismo (Figura $3 \mathrm{~b})$. Portanto, o deslocamento relativo $\left(u_{r}\right)$ produzido pela excitação sísmica é o mesmo que ocorre quando o sistema é considerado com uma base fixa e submetido a uma força externa dada por $-m \ddot{u}_{g}(t)$.

Para um sistema de parâmetros distribuídos a força equivalente pode ser obtida de forma análoga, com aplicação do princípio dos trabalhos virtuais. Então:

$$
\tilde{F}(t)=-\ddot{u}_{g}(t) \int_{0}^{H} \mu(y) \phi(y) d y=-\ddot{u}_{g}(t) \tilde{L}
$$


onde $\tilde{L}$ representa o fator de excitação sísmico, que indica a participação do sismo na reposta de uma dada forma modal $\phi(y)$. Assim, a equação de equilíbrio dinâmico não-amortecida de um modo arbitrário será dada por:

$$
\widetilde{M} \ddot{X}(t)+\widetilde{K} X(t)=-\ddot{u}_{g}(t) \tilde{L}
$$

As expressões anteriores são válidas para um sistema desacoplado. Quando o problema envolve dois domínios (fluido e estrutura) considerações especiais devem ser feitas, pois o movimento translacional produz um campo de pressões diferente do produzido pelo movimento relativo (vide Figura 2). Assim, é comum encontrar na literatura a divisão em: pressão de movimento de corpo rígido e pressão associada à deformada modal (FERC, 2002). Uma interpretação simples para este fenômeno pode ser estabelecida definindo duas forças inerciais produzidas pelo fluido: $\mathrm{f}_{r} \ddot{u}_{r}(t)$ e $\mathrm{F}_{g} \ddot{u}_{g}(t)$, proporcionais às acelerações relativa $\left(\ddot{u}_{r}\right)$ e do solo $\left(\ddot{u}_{g}\right)$. Assim, a equação de equilíbrio dinâmico de um sistema de um grau de liberdade não-amortecido será dada por:

$$
m \ddot{u}_{t}(t)+K u_{r}(t)=-\mathfrak{f}_{r} \ddot{u}_{r}(t)-\mathfrak{f}_{g} \ddot{u}_{g}(t)
$$

Substituindo $\ddot{u}_{t}(t)=\ddot{u}_{g}(t)+\ddot{u}_{r}(t)$ nesta última expressão resulta:

$$
\left[m+\mathfrak{f}_{r}\right] \ddot{u}_{r}(t)+K u_{r}(t)=-\ddot{u}_{g}(t)\left[m+\mathfrak{f}_{g}\right]
$$

A análise de (15) revela a presença de forças inerciais produzidas pelo fluido em ambos os lados desta expressão. É importante notar que a massa adicionada ao lado esquerdo $\left({ }_{F}\right)$ é diferente da associada ao lado direito $\left({ }^{F_{g}}\right)$, já que a primeira encontra-se relacionada ao movimento relativo do sistema, ao passo que a segunda está associada ao movimento translacional. Adicionalmente, observa-se que os efeitos inerciais do fluido continuam presentes mesmo quando a excitação do solo é nula (no caso de vibração de um sistema acoplado com base fixa, por exemplo). A Figura 4 ilustra um esquema com as forças equivalentes do problema acoplado. 


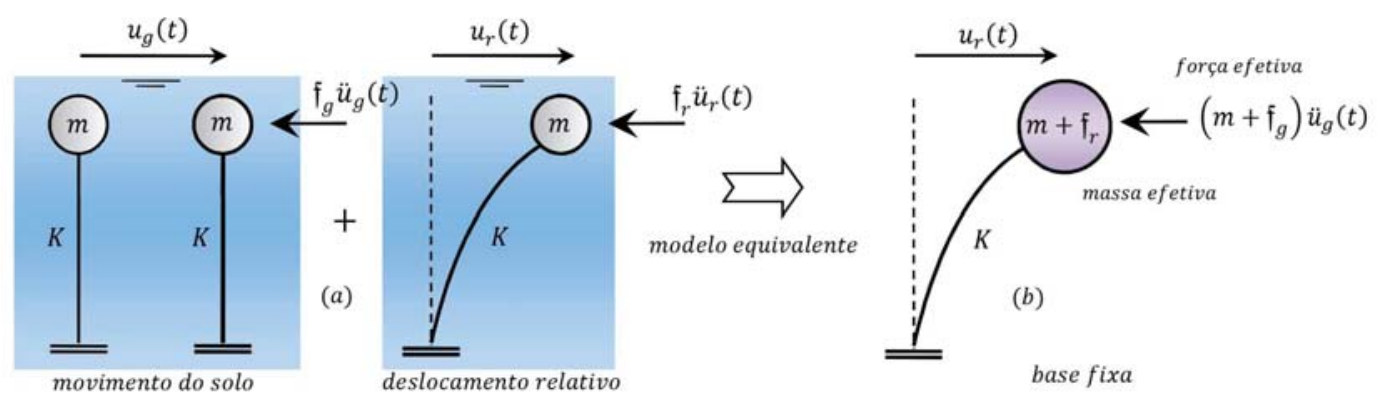

Figura 4: Excitação sísmica de um sistema de 1 grau de liberdade. Sistema acoplado (a), modelo equivalente (b)

O mesmo raciocínio pode ser empregado em um sistema com parâmetros distribuídos. A identificação dos termos inerciais é facilmente obtida pelo esquema indicado na Figura 4. Neste caso, a equação de movimento não-amortecida em termos de uma coordenada generalizada $X(t)$ é dada por:

$$
\left(\widetilde{M}+\widetilde{M}_{\text {fluido }}\right) \ddot{X}(t)+\widetilde{K} X(t)=-\ddot{u}_{g}(t)[\widetilde{L}+\widetilde{N}]
$$

Esta última expressão revela a influência do reservatório tanto na massa do sistema (Figura 5b), definida por (1), como na parcela de excitação sísmica efetiva. Neste último caso, o problema pode ser interpretado como a aceleração de um bloco barragem-reservatório-fundação com uma intensidade idêntica a aceleração do solo (Figura 5a). Assim, o termo $\widetilde{N}$ corresponde aos efeitos inerciais do reservatório em contato com uma parede rígida acelerada uniformemente e pode ser representado adequadamente pelo caso (IR), indicado na Figura 2. Então, de forma análoga a (12):

$$
\widetilde{N}=\int_{0}^{H} \mathfrak{f}_{g}(y) \phi(y) d y
$$

onde:

$$
\mathfrak{f}_{g}(y)=\frac{P(0, y, \omega)}{\bar{A}}=\frac{2 \rho_{f}}{H} \sum_{n=1}^{\infty} \frac{(-1)^{n+1}}{\kappa_{n}{ }^{2}} \cos \left(\kappa_{n} y\right) \quad \rightarrow I R\{\phi(y)=1, c \rightarrow \infty\}
$$



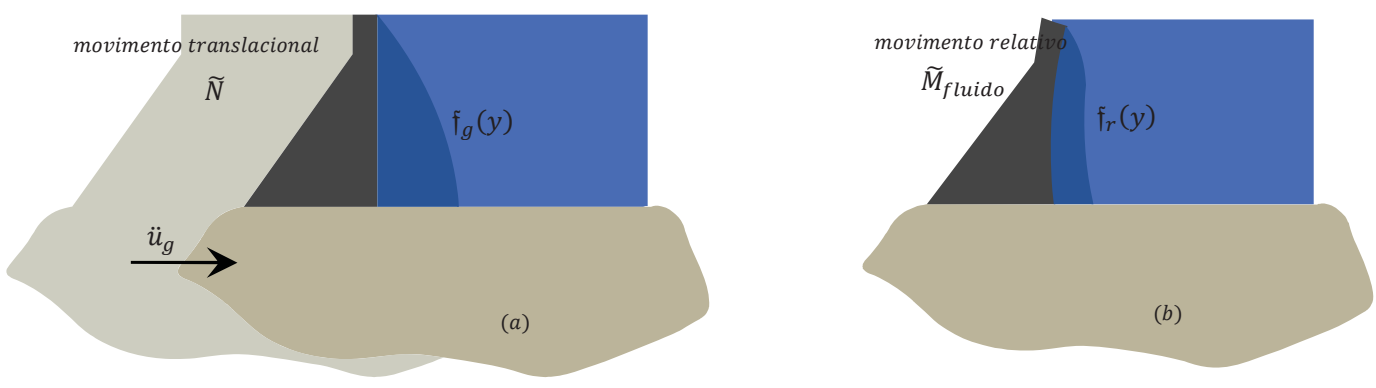

Figura 5: Componentes translacionais (a) e relativas (b) produzidas pela interação com o fluido

\section{Avaliação da resposta dinâmica e análise estática equivalente}

A avaliação da resposta dinâmica de (13) ou (16) frente aos registros de uma excitação sísmica pode ser conduzida com uma rotina de integração numérica (embora para funções mais simples uma avaliação analítica seja possível). Neste trabalho foi empregado um algoritmo de Runge-Kutta de quarta ordem, desenvolvido em linguagem Fortran 90. Resultados imediatos de deslocamentos relativos são obtidos, sendo posteriormente aplicados em uma análise de tensões da barragem.

É importante introduzir o conceito de forças laterais equivalentes $\left(q_{\varepsilon}\right)$, cujos efeitos produzem deformações semelhantes às produzidas pelo sismo (Clough, 1993 e Chopra, 2001). Da teoria clássica de dinâmica das estruturas resulta a expressão (19), relacionada a uma análise sem a influência do reservatório. Assim:

$$
q_{e}(y, t)=m_{s}(y) \phi(y)\left[\omega^{2} \frac{\tilde{L}}{\widetilde{M}} \mathcal{D}(t)\right]
$$

onde $m_{s}(y)$, define a distribuição de massa ao longo da altura da barragem e $\mathcal{D}(t)$ indica o deslocamento relativo de um sistema de um grau de liberdade com freqüência $\varpi$ (no vácuo). O termo entre colchetes é denominado pseudo-aceleração, pois corresponde à aceleração que efetivamente produz deformações na estrutura, tendo significado distinto da aceleração total (sendo idêntico apenas no caso de sistemas não-amortecidos). Esta última expressão permite uma análise estática equivalente (utilizando teoria de vigas ou aplicando cargas estáticas em um modelo numérico, por exemplo) da geometria da barragem submetida aos maiores efeitos produzidos pelo sismo $\left(\mathcal{D}_{\text {máx }}\right.$, obtido em uma análise espectral).

Para análises com a inclusão dos efeitos hidrodinâmicos resulta a seguinte expressão (CHOPRA, 1978): 


$$
q_{r}(y, t)=\left\{m_{s}(y) \phi(y)+m_{f}(y)\right\}\left[\omega^{2} \frac{\tilde{L}_{r}}{\widetilde{M}_{r}} \mathcal{D}(t)\right]
$$

onde $\widetilde{L}_{r}=\widetilde{L}+\widetilde{N}$ e $\widetilde{M}_{r}=\widetilde{M}+\widetilde{M}_{\text {fluido }}$ indicam, respectivamente, os termos de excitação e massa generalizadas incluindo os efeitos do reservatório. $\mathrm{E} \mathcal{D}(t)$ corresponde ao deslocamento relativo de um sistema de um grau de liberdade com frequência $\omega$ (incluindo efeitos hidrodinâmicos). Adicionalmente, surge o parâmetro $m_{f}(y)$,, relacionado ao efeito inercial do fluido ao longo da altura da barragem, definido por:

$$
m_{f}(y)=\frac{P(0, y, \omega)}{\bar{A}} \quad \rightarrow C F
$$

As expressões (19) e (20) envolvem termos definidos em seções anteriores e podem ser aplicadas sem maiores dificuldades na construção de um carregamento equivalente e posterior solução de um problema estático. Na equação (21) atenção especial deve ser dada ao modelo de pressão hidrodinâmica, tomado como compressível e flexível (CF).

\section{Exemplo de aplicação / Estudo de Caso}

Um perfil típico com geometria ilustrada na Figura 6a foi avaliado com o procedimento proposto. As análises envolvem tanto a resposta dinâmica, como o campo de tensões produzido em um instante de tempo arbitrário. Três registros sísmicos com características distintas foram aplicados e os resultados comparados aos obtidos em uma solução numérica acoplada, cujo modelo encontra-se indicado na Figura 6b. Para a estrutura foi adotado um módulo de elasticidade $E=25 \cdot 10^{9} \mathrm{~Pa}$ e uma densidade $\rho_{s}=2400 \mathrm{~kg} / \mathrm{m}^{3}$. Para o fluido admite-se uma densidade $\rho_{f}=1000 \mathrm{~kg} / \mathrm{m}^{3}$ e uma velocidade de propagação do $\operatorname{som} c=1500 \mathrm{~m} / \mathrm{s}$.

O modelo numérico foi elaborado com auxílio do programa ANSYS v. 11, empregando elementos de estado plano de deformação, com quatro nós por elemento na discretização da estrutura. Os nós da base do modelo foram engastados. Para o fluido foram utilizados elementos acústicos planos de quatro nós. Uma relação $L / H=3$ foi adotada na discretização do reservatório, com condições de contorno de pressão nula na extremidade $x=L$ e na superfície $y=H$. 

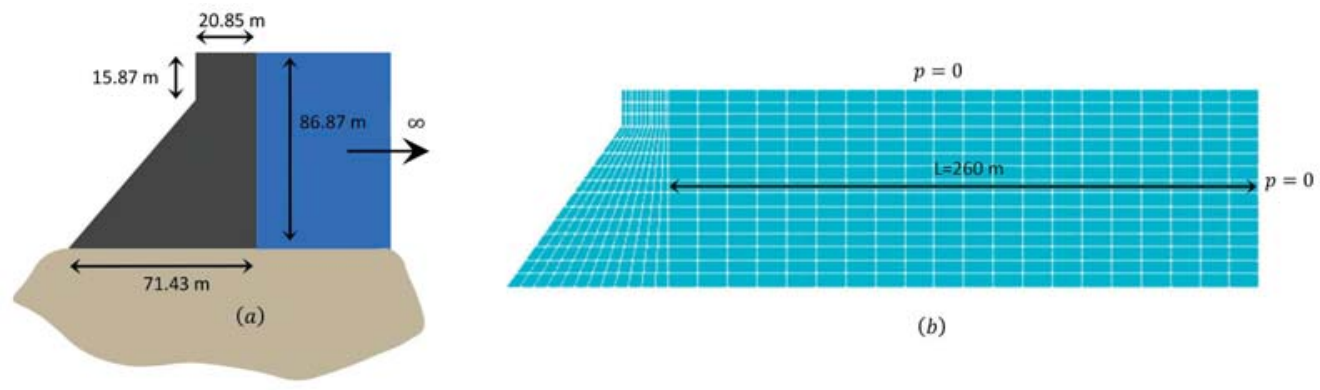

(b)

Figura 6: Geometria da barragem (a) e malha em elementos finitos (b)

A seguir são apresentadas as principais etapas e resultados obtidos.

\subsection{Análise modal da estrutura desacoplada}

De acordo com o esquema apresentado na Figura 1 são obtidos, em uma análise modal auxiliar, três parâmetros relacionados ao modo fundamental, indicados na Tabela 1. Neste caso, a freqüência fundamental tem valor igual a $26.846 \mathrm{rad} / \mathrm{s}$.

Tabela 1: Parâmetros generalizados - análise modal (modo fundamental)

\begin{tabular}{ccc}
\hline $\begin{array}{c}\text { Massa Generalizada }\left(\frac{N s^{2}}{m}\right) \\
\widetilde{M}\end{array}$ & Rigidez Generalizada $\left(\frac{N}{m}\right)$ & Fator de Participação Sísmico $\left(\frac{N s^{2}}{m}\right)$ \\
\hline $\mathbf{1 . 0 6 3 8} \cdot \mathbf{1 0}^{\mathbf{6}}$ & $\widetilde{\boldsymbol{K}}$ & $\widetilde{\boldsymbol{L}}$ \\
\hline
\end{tabular}

Uma função de interpolação é estabelecida para os deslocamentos horizontais dos nós da interface resultando na seguinte expressão:

$$
\phi(y)=-0.7663\left(\frac{y}{H}\right)^{4}+1.5247\left(\frac{y}{H}\right)^{3}-0.1043\left(\frac{y}{H}\right)^{2}+0.3482\left(\frac{y}{H}\right)-0.0003
$$

\subsection{Solução da equação de frequências (frequência fundamental acoplada)}

A forma geral da equação de frequências é indicada na expressão abaixo, que é resolvida com a inclusão dos parâmetros generalizados obtidos na análise modal auxiliar.

$$
\widetilde{K}-\omega^{2}\left[\widetilde{M}+\int_{0}^{H} \frac{P(0, y, \omega)}{\bar{A}} \phi(y) d y\right]=0
$$


O termo $P(0, y, \omega)$ depende das considerações adotadas para as pressões hidrodinâmicas, esquematizadas na Figura 2. A Tabela 2 ilustra os resultados da avaliação desta última expressão para os casos CF e IF.

Tabela 2: Resultados da equação de freqüências (modo fundamental acoplado)

\begin{tabular}{cccc}
\hline \multicolumn{2}{c}{ Compressível e Flexível (CF) } & \multicolumn{2}{c}{ Incompressível e Flexível (IF) } \\
\hline Número de termos na série $(n)$ & $\omega(\mathrm{rad} / \mathrm{s})$ & Número de termos na série & $\omega(\mathrm{rad} / \mathrm{s})$ \\
3 & 22.108 & 3 & 23.222 \\
5 & 22.008 & 5 & 23.083 \\
10 & $\underline{21.966}$ & 10 & 23.025 \\
\hline
\end{tabular}

A massa generalizada produzida pelo fluido $\left(\widetilde{M}_{\text {fluido }}\right)$ é obtida de forma imediata com o rearranjo de (23). Assim, para o caso $\mathrm{CF}$, $\operatorname{com} n=10$, resulta:

$$
\omega^{2}=\frac{\widetilde{K}}{\widetilde{M}+\widetilde{M}_{\text {fluido }}} \quad \therefore \quad \widetilde{M}_{\text {fluido }} \cong 0.5203 \cdot 10^{6} N s^{2} / m
$$

\subsection{Análise modal do modelo numérico acoplado}

Resultados de uma análise modal do modelo indicado na Figura 6b são apresentados na Tabela 3, onde são evidenciados os modos de vibração e as frequências associadas. A frequência fundamental obtida no item anterior (caso CF) está de acordo com o valor indicado nesta tabela, com um erro relativo de aproximadamente $1 \%$.

Tabela 3: Resultados obtidos com o modelo numérico

\begin{tabular}{cccc}
\hline Modo & $\omega(\mathrm{rad} / \mathrm{s})$ & Deformada modal \\
\hline 1 & 30.014 & \\
2 & 38.734 & \\
\hline
\end{tabular}




\subsection{Equação de movimento da estrutura acoplada}

Os parâmetros generalizados da Tabela 1 e as expressões (1) e (17) são aplicados em (16) para construção da equação de movimento do modo fundamental da estrutura acoplada. Assim:

$$
1.58 \cdot 10^{6} \ddot{X}(t)+7.67 \cdot 10^{8} X(t)=-\ddot{u}_{g}(t) 3.17 \cdot 10^{6} \quad \rightarrow \text { acoplado }
$$

onde $X(t)$ corresponde ao deslocamento no paramento de montante de um ponto localizado na crista da barragem.

A última equação permite a avaliação de deslocamentos e tensões para uma excitação arbitrária do solo $\ddot{u}_{g}(t)$. A seguir são apresentados resultados envolvendo registros sísmicos.

\subsection{Resposta da estrutura acoplada para excitações de origem sísmica}

Três registros sísmicos distintos e seus respectivos espectros de freqüências são apresentados nas Figura 7, 8 e 9. Os dados correspondem a eventos reais, relacionados aos sismos de Imperial Valley (1940, estação El Centro,componente norte-sul), Hollister (1974, estação City Hall) e Loma Prieta (1989, estação Emeryville).
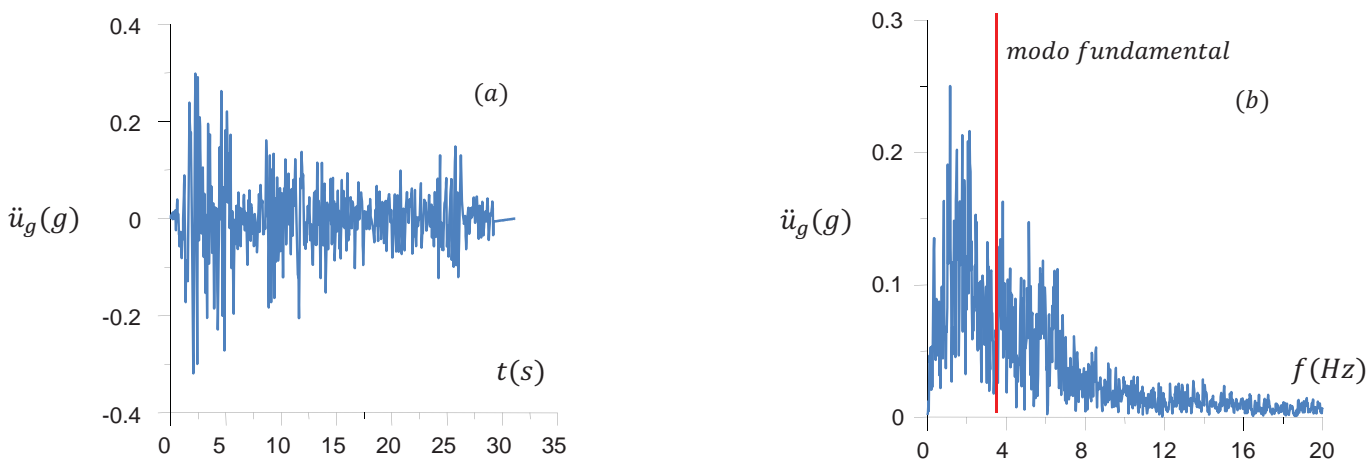

Figura 7: Registro de movimento do solo - Imperial Valley (a), espectro de frequências (b) 

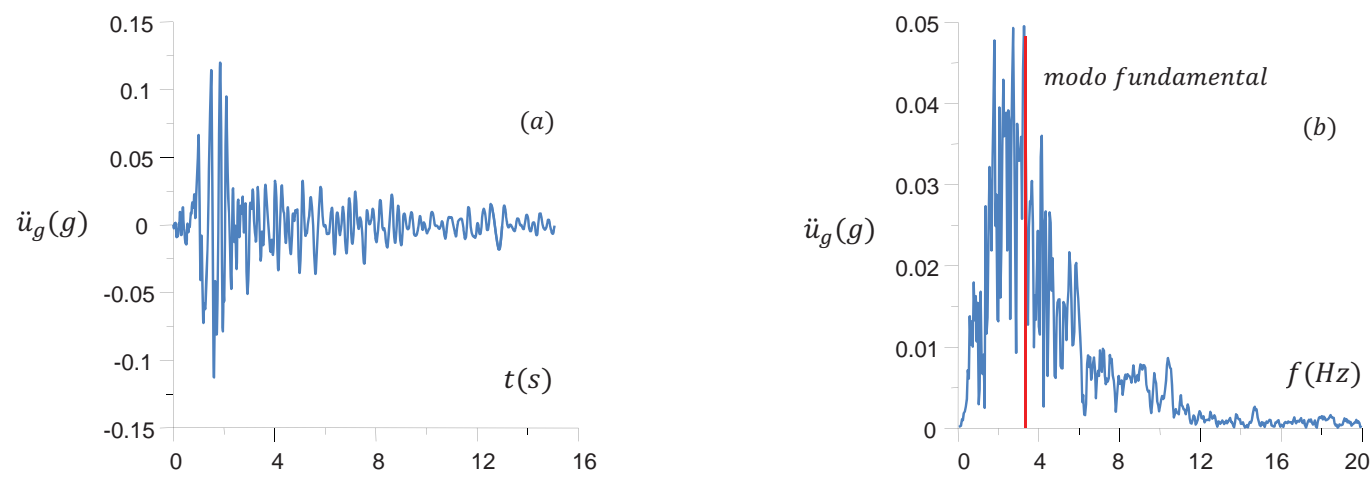

Figura 8: Registro de movimento do solo - Hollister (a), espectro de frequências (b)
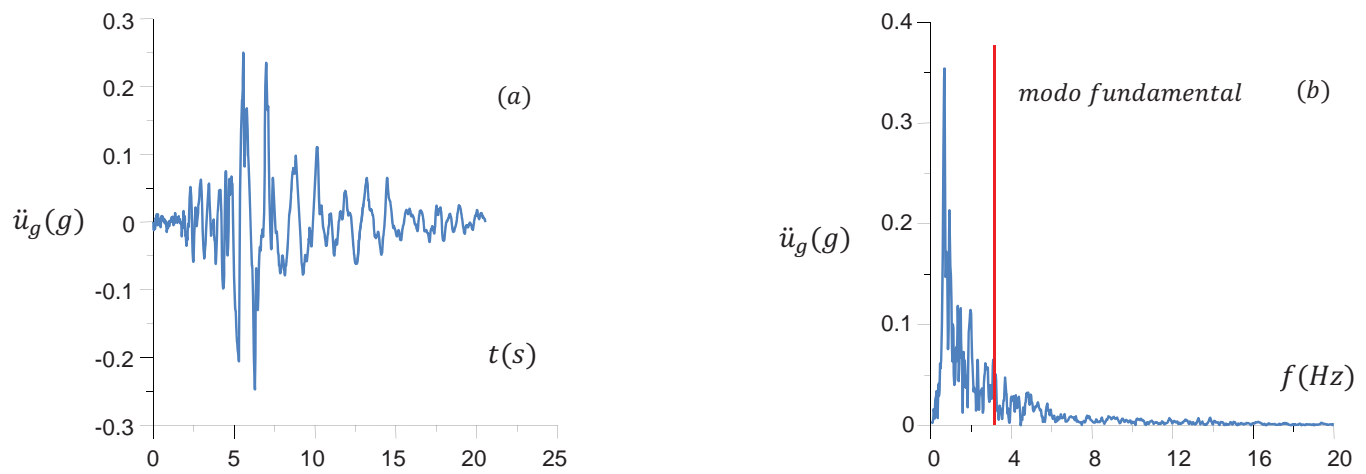

Figura 9: Registro de movimento do solo - Loma Prieta (a), espectro de frequências (b)

As Figuras 7b, 8b e 9b refletem uma particularidade: amplitudes dominantes nas frequências mais baixas. Este aspecto justifica uma participação dos modos inicias na resposta dinâmica. Curvas comparativas entre a resposta numérica do sistema acoplado (referente aos deslocamentos relativos da crista da barragem, $X$ ) e a avaliação da expressão (25) em uma rotina de integração numérica (Runge-Kutta) são apresentadas na Figura 10, relativas ao sismo de Imperial Valley. Observa-se uma excelente aproximação em instantes iniciais (Figura 10a), com erros cumulativos à medida que o intervalo de tempo é ampliado (Figura 10b). 

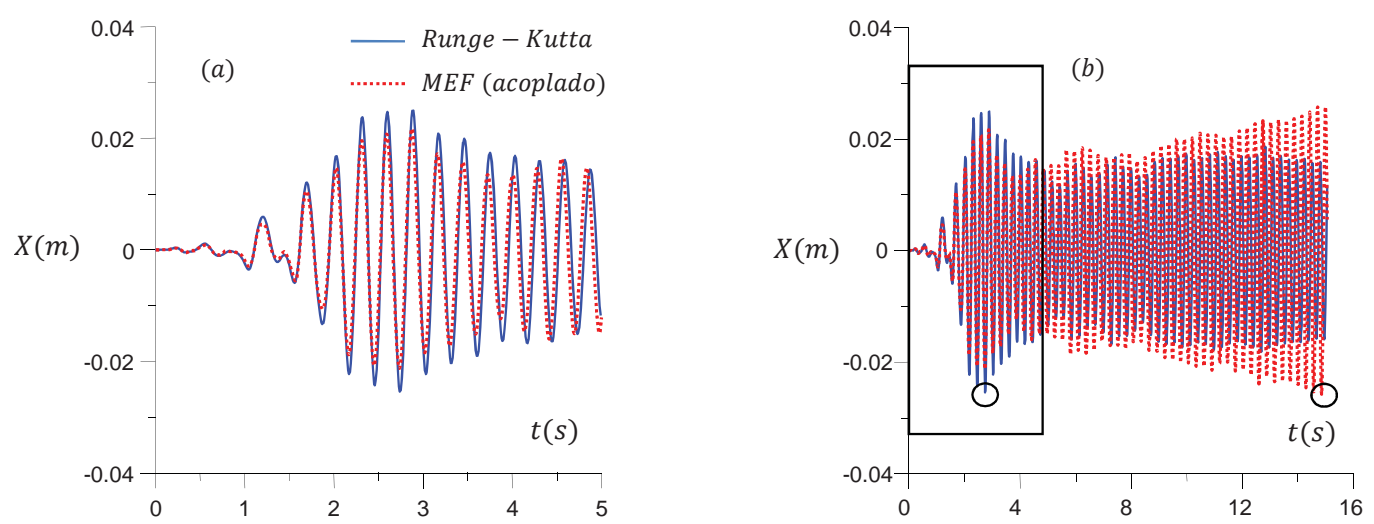

Figura 10: Resposta de deslocamentos relativos - Imperial Valley. Janela de tempo (a), Registro completo (b)

Na Figura 10b também são indicados os pontos de resposta máxima absoluta de deslocamentos relativos às duas soluções. Para a solução proposta o valor máximo de $X=-0.063$ ocorre em $t=15.07 \mathrm{~s}$. Na solução acoplada o valor máximo $X=0.075 \mathrm{~m}$ ocorre em $t=21.74 \mathrm{~s}$. Neste caso específico a solução proposta é aproximadamente $84 \%$ do deslocamento obtido com a solução acoplada. Estas diferenças ocorrem devido aos erros cumulativos produzidos ao longo do intervalo de duração do evento e são importantes na construção do carregamento equivalente.

As soluções de deslocamentos relativos para os eventos de Hollister e Loma Prieta são ilustradas, respectivamente, nas Figuras 11 e 12.
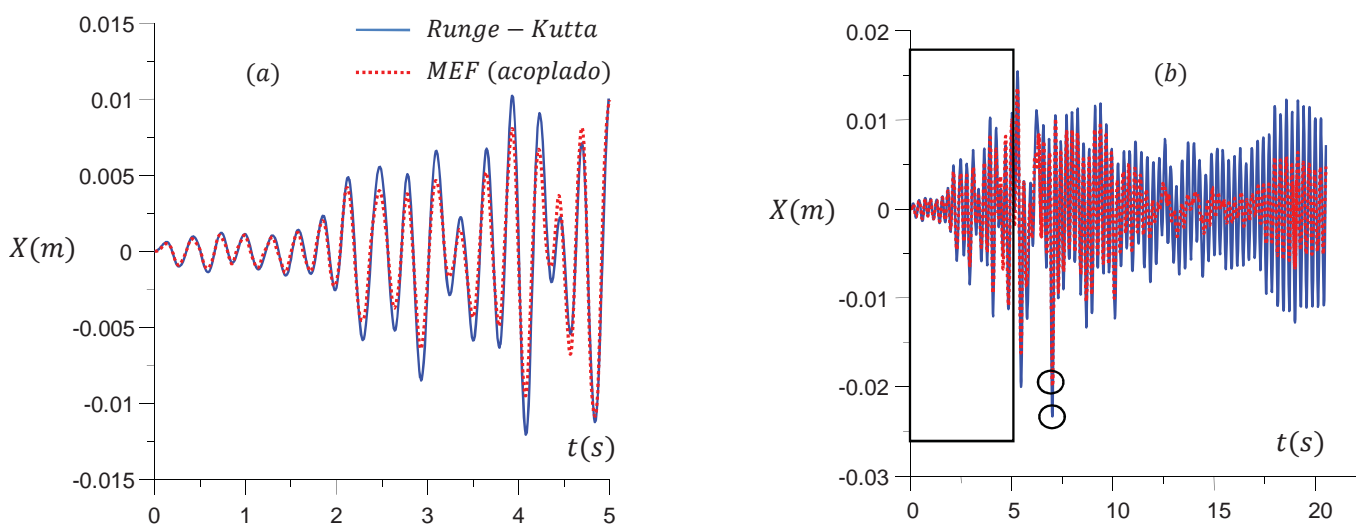

Figura 11: Resposta de deslocamentos relativos - Hollister. Janela de tempo (a), Registro completo (b) 

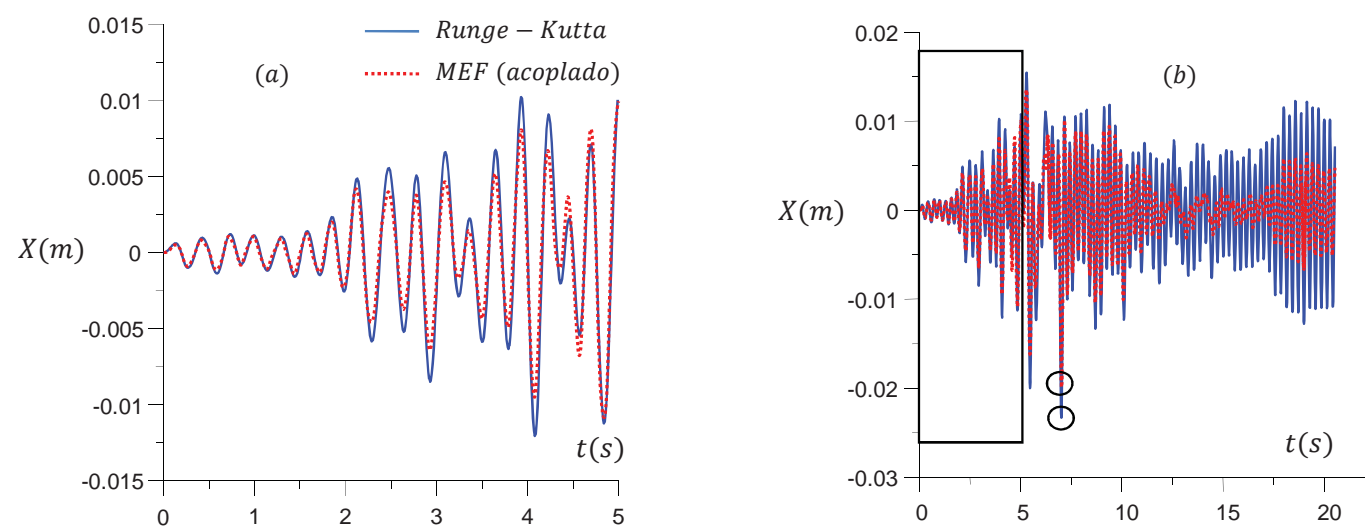

Figura 12: Resposta de deslocamentos relativos - Loma Prieta. Janela de tempo (a), Registro completo (b)

\subsection{Avaliação das tensões máximas desenvolvidas na barragem}

A avaliação de tensões na solução numérica acoplada é obtida de forma imediata, com a investigação dos resultados no instante de deslocamento relativo máximo absoluto. No caso do procedimento proposto, uma análise no domínio do tempo torna-se desnecessária, já que é mais prático o emprego de um espectro de deslocamentos, conforme ilustrado na Figura 13, que indica os espectros obtidos com o auxílio do software SeismoSignal v. 3.3.0, correspondentes a um sistema não-amortecido. Neste caso, os valores de interesse (indicados na figura) são relativos ao modo fundamental acoplado, com período de vibração $T=0.286 \mathrm{~s}(22 \mathrm{rad} / \mathrm{s})$.
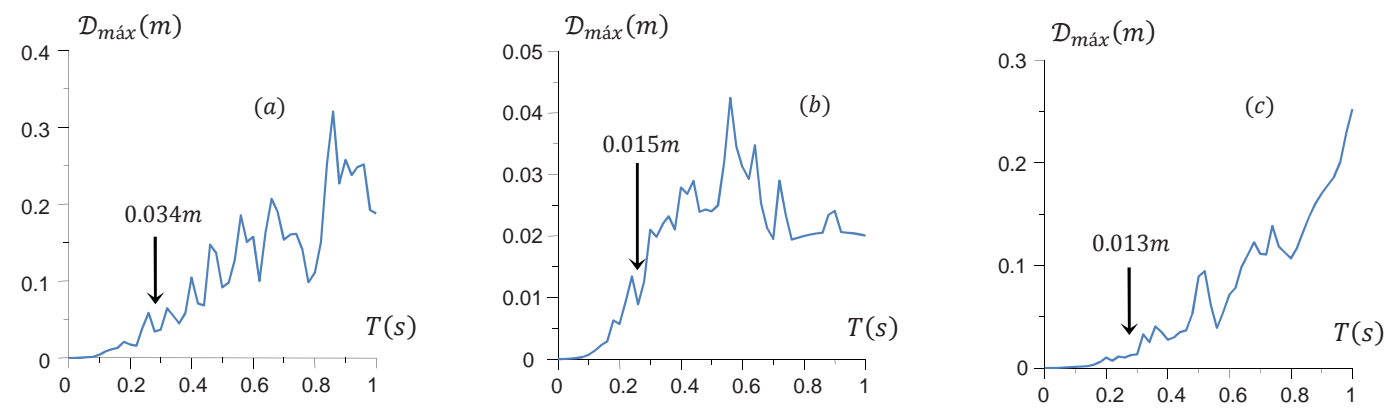

Figura 13: Espectro de deslocamentos: Imperial Valley (a), Hollister (b) e Loma Prieta (c) 
Conforme comentado anteriormente, o carregamento estático é obtido com aplicação direta de (20) ao longo do paramento de montante da barragem. Assim, a análise dinâmica de um problema acoplado é reduzida a uma análise estática equivalente da geometria da estrutura. Na Tabela 4 são apresentados os resultados de tensões obtidos com o procedimento proposto e com a análise acoplada em elementos finitos.

Tabela 4: Resultados de tensões principais (Método dos Elementos Finitos e procedimento proposto)

\begin{tabular}{ccc}
\hline Sismo & Tensões principais máximas (kPa) \\
\hline Imperial Valley & Procedimento proposto \\
\hline Hollister & & \\
\hline Loma Prieta & & \\
\hline
\end{tabular}

61 


\section{Conclusões}

Um procedimento semi-analítico foi apresentado para avaliação da resposta dinâmica de barragens de gravidade de concreto submetidas a excitações sísmicas. $O$ processo é simples e baseado em uma análise modal auxiliar da geometria da barragem em um código de elementos finitos convencional. Uma equação de movimento do modo fundamental permite a análise da resposta de deslocamentos do sistema em coordenadas generalizadas. Os gráficos indicados nas Figuras 7, 8 e 9 revelam uma excelente aproximação, nos instantes iniciais da excitação, frente a resultados numéricos acoplados. Erros cumulativos em instantes posteriores levam a diferenças significativas entre as respostas. De maneira geral, as amplitudes absolutas apresentam grande aproximação. Esta semelhança permite uma avaliação de tensões por meio de um espectro de deslocamentos, conforme ilustrado na Figura 13. Um carregamento equivalente é construído em função do maior deslocamento espectral e em seguida aplicado em uma análise estática em elementos finitos da geometria da barragem. A comparação entre os resultados deste procedimento e uma análise numérica acoplada é ilustrada na Tabela 4, que revela resultados satisfatórios para a distribuição de tensões no maciço. É evidente que a aproximação é função direta da diferença relativa entre os deslocamentos do modelo acoplado e o obtido com a solução proposta. Nos eventos de Hollister e Loma Prieta, por exemplo, a aproximação foi excelente. Por outro lado, no sismo de Imperial Valley os resultados foram apenas satisfatórios.

Dois pontos de destaque merecem atenção especial no procedimento proposto. $\mathrm{O}$ primeiro é a avaliação da distribuição de tensões em códigos computacionais de elementos finitos envolvendo apenas um dos domínios: a estrutura. Neste caso, o estudo de um problema relativamente complexo passa a ser realizado em programas convencionais, com limitações em análises acopladas. Este artifício torna universal a análise da interação barragem-reservatório em escritórios de projetos de barragens onde um pacote básico de elementos finitos esteja disponível. O segundo é a construção de equações de referência para validação dos resultados de deslocamentos obtidos em modelos acoplados. Embora a aproximação ocorra apenas em instantes iniciais, correções de freqüências podem ser aplicadas, modificando parâmetros generalizados para uma melhor aproximação entre freqüências semi-analítica e numérica. Assim, é desenvolvida uma ferramenta auxiliar para verificação de testes de convergência em modelos numéricos. A principal limitação surge em decorrência da utilização de um único modo na construção da resposta dinâmica. Entretanto, os resultados anteriores revelam que a aproximação é satisfatória, viabilizando análises preliminares deste tipo de estrutura. 


\section{Referências}

ANSYS Multiphysics v.11. Ansys Inc.

CHOPRA, A. K., 1970. "Earthquake Response of Concrete Gravity Dams". In: Report No EERC 70-1, Earthquake Engineering Research Center, University of California - Berkeley.

, 1978, "Earthquake Resistant Design of Concrete Gravity Dams", Journal of the Structural Division, ASCE, vol. 104, n. ST6, pp. 953-971.

CHOPRA, A. K. "Dynamics of Structures: Theory and Applications to Earthquake Engineering". 2a ed., Prentice Hall, New Jersey, 2001.

CLOUGH, R. W.; Penzien, J. “Dynamics of Structures”. 2ed., McGraw-Hill, Inc., New York, 1993.

FERC (Federal Energy Regulatory Commission), 2002. "Chapter III Gravity Dams”. In: Federal Energy Regulatory Commission, Office of Hydropower Licensing. Engineering guidelines for evaluation of hydropower projects. Washington.

FENVES, G. F., Chopra, A. K., 1985, "Effects of Reservoir Bottom Absorption and Dam-Water-Foundation Rock Interaction on Frequency Response Functions for Concrete Gravity Dams." Earthquake Engineering and Structural Dynamics, vol. 13, pp. 13-31.

LEE, G. C.; Tsai, C. S., 1991. "Time domain analyses of dam reservoir system. I: Exact solution”. In: Journal of Engineering Mechanics, ASCE, vol. 117, no. 9, p. 1990-2006.

MIQUEL, B., Bouaanani, N., 2010. "Simplified evaluation of the vibration period and seismic response of gravity dam-water systems”. Engineering Structures, Vol. 32, pp. 2488-2502.

RIBEIRO, P. M. V., Melo, C. A. E., Pedroso, L. J. 2009 "Analytical Solution of Dam-Reservoir Interaction in the Fundamental Mode Shape". In: M. Alves; H. S. da Costa Mattos. (Org.). Solid Mechanics in Brazil - 2009. São Paulo: ABCM - Associação Brasileira de Engenharia e Ciências Mecânicas, 2009, v. , p. 445-473.

RIBEIRO, P. M. V., 2010, "Soluções Analíticas para Cavidades Acústicas Bidimensionais com Aplicação ao Estudo da Interação Dinâmica Barragem-Reservatório", Tese de Doutorado em Estruturas e Construção Civil, Publicação E.TD-004A/10, Departamento de Engenharia Civil e Ambiental, Universidade de Brasília, Brasília, DF, 306 p.

Seismosignal v. 3.3.0. Seismosoft Ltd.

WESTERGAARD, H. M., 1933. "Water pressures on dams during earthquakes". In: Transactions of the American Society of Civil Engineers, v. 98, p. 418-433. 


\section{Relação de símbolos e notações}

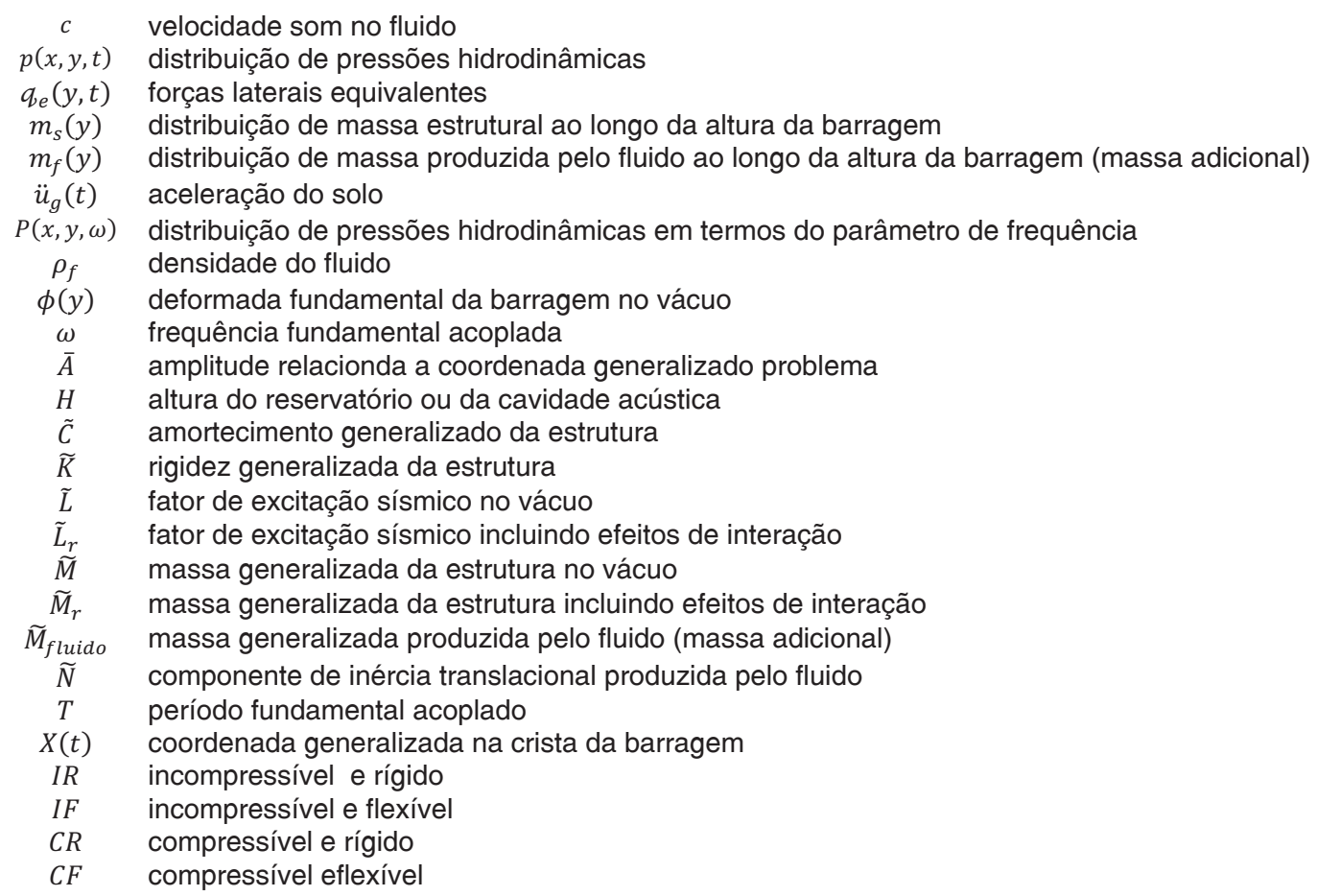




\title{
A simplified procedure for dynamic analyses of concrete gravity dams
}

\begin{abstract}
In a previous work a simplified procedure was developed for the analysis of dam-reservoir interaction considering only the contribution of the fundamental mode, with solution of a frequency equation and subsequent construction of an equivalent numerical model. This paper presents a sequence of this research by exploring the highlights of the proposed methodology, such as variation of the excitation source, the influence of hydrodynamic pressures model, contribution of higher modes on the dynamic response and evaluation of stresses along the monolith. At the end, an application example will be given along with a subsequent discussion of the advantages and limitations of the proposed procedure.
\end{abstract}

Keywords: Dams. Reservoir. Fluid-Structure Interaction. Semi-Analytical. Dynamic.

\section{Introdution}

Dynamic analyses of dams subjected to interaction effects of the adjacent reservoir is a subject of great interest in seismically active regions. Numerical solutions involving both domains (fluid and structure) are generally expensive in terms of computational processing time. Additionally, they are limited to specific computer codes which allow the analysis of multiphysic problems. The three-dimensional geometry of the structure can be reduced to a specific two-dimensional case (in gravity dams, for example). Still, there are numerous possibilities for the typical geometry adopted, turning an entirely analytical solution practically impossible, or applied only to a few specific cases, as shown by Tsai and Lee (1991). An alternative arises with the use of intermediate solutions, using the precision of analytical solutions coupled with the wide range application of numerical methods.

The problem of dam-reservoir interaction has been analyzed by several authors. The vast majority of work basically follows the same line of development of classic articles produced by Chopra $(1970,1978)$ and his co-workers (Fenves and Chopra, 1985). Since the pioneering work of Westergaard (1933), the importance of including the effects of dynamic interaction with the fluid was revealed. The dam, which was originally supposed rigid, became flexible. In a next step the effects of fluid compressibility were included in the analysis, with dependence on the vibration frequency of the dam-reservoir system. Numerous practical procedures were developed considering the vibration period as a starting point for evaluation of interaction effects. The foundation absorption influence was added at a later stage. The common point in all these previous articles is exactly the presence of project abaci, based on a standard dam geometry, which allows the relation of the in-vacuum and coupled periods of vibration. 
Since interaction effects are dependent of the coupled frequency, this step proves to be the most important of the analysis, with recent interest of researchers in search of semi-analytical solutions for the coupled vibration period in arbitrary dam geometries, avoiding the use simplifications inherent to the previous procedures. The current limitation is that even the most recent proposals for semi-analytical procedures, such as Bouanaani and Miquel (2010) and Ribeiro et al. (2009) are still limited to dam-reservoir interaction effects, neglecting any form of foundation interaction.

This paper presents an effective strategy in the elimination of the reservoir domain, replacing it with equivalent effects on the fluid-structure interface. Thus, the problem can be simplified to the solution of a single dynamic equilibrium equation. It is evident that the proposed solution is only valid for the fundamental mode shape of dam-reservoir systems, with all generalized parameters consistent with the fundamental mode. This is the main limitation of the proposed procedure.

\section{Conclusions}

A semi-analytical procedure was presented for evaluation of the dynamic response of concrete gravity dams subjected to seismic excitations. The process is simple and based on in-vacuum analysis of the dam geometry in a conventional finite element code. An equation of motion of the fundamental mode allows the analysis of response displacements of the system in terms of generalized coordinates. The graphs shown in Figures 7, 8 and 9 show an excellent agreement in the short range when compared to numerical results. Cumulative errors in subsequent instants lead to significant differences between the responses at long range analyses. In general, absolute amplitude approximation is achieved with the current procedure. This similarity allows a stress analysis evaluation, as illustrated in Figure 13. An equivalent load is built according to the largest spectral displacement and then applied in a static finite element analysis of the dam geometry. A comparison of the results of this procedure and the coupled numerical analysis results is shown in Table 4, which presents satisfactory stress distribution in the current method.

Two points deserve special attention in the proposed procedure. The first is evaluation of stress distribution in finite element computer codes involving only one domain: the structure. In this case, the study of a relatively complex problem can be carried out in conventional programs, avoiding the need of multiphysic packages. This enables universal analysis of dam-reservoir interaction in project offices where a basic package of finite element code is available. The second is the construction of reference equations for benchmark tests in coupled models. Although convergence occurs only in the short range, corrections can be applied by modifying generalized parameters or time steps in the Runge-Kutta procedure to a better approximation between semi-analytical and numerical results. The main limitation arises due to the use of a single mode in the construction of the dynamic response. However, previous results show that the approach is satisfactory, enabling preliminary analyses of this type of structure. 